\title{
METANALISE: O VALOR DA ANGIOTOMOGRAFIA NA AVALIAÇÃO VASCULAR PRÉ TRANSPLANTE RENAL
}

\section{Marcelo L. Lima, Graziele Ambrosio*, Renan J. Rigonato}

\section{Resumo}

A angiotomografia, método não invasivo capaz de detectar com precisão calcificações arteriais, pode ser utilizada para previnir complicações pós tranplante renal devido a calcificação de artérias ilíacas.O objetivo desse trabalho é verificar o valor da angiotomografia como método diagnóstico de calcificações e doenças arteriais periféricas e seu valor prognóstico em candidatos a terapia substitutiva renal, atraves da análise de estudos primários de pacientes submetidos a este exame.Como metodologia foi realizada busca literária em bases de dados eletrônicos (PubMED, LILACS, GOOGLE SCHOLAR)que resultou na analise de 802 pacientes submetidos a CT (angiotomografia).Todos os estudos demonstraram alta acurácia no diagnóstico da calcificação por CT,e desfechos relacionados como alteração do local de anastomose $(25,2 \%)$,suspensão do transplante( $7,6 \%)$ e perda do enxerto $(6,8 \%)$.

\section{Palavras-chave:}

Angiotomografia, calcificação arterial, transplante renal .

\section{Introdução}

A calcificação arterial, que possui alta incidência em pacientes renais crônicos, é um dos principais fatores de risco para complicações cirúrgicas imediatas e tardias no pós-operatório do transplante renal.

Os métodos radiológicos de avaliação da calcificação dos vasos periféricos ainda são limitados. A angiotomografia das artérias ilíacas, local do implante renal, pode mostrar com precisão as doenças arteriais, incluindo a localização e a extensão da calcificação arterial, além do calibre arterial, o que permitiria um melhor planejamento cirúrgico. O objetivo desse trabalho foi verificar 0 valor da Angiotomografia computadorizada como método diagnóstico de calcificações e doenças arteriais periféricas e seu valor prognóstico em pacientes candidatos a terapia substitutiva renal, através da análise de estudos primários de pacientes submetidos a este exame, obtidos por meio de busca literária em bases de dados eletrônicos (PubMED, LILACS, GOOGLE SCHOLAR ).

\section{Resultados e Discussão}

Foram incluídos estudos primários que avaliaram o valor diagnóstico e prognóstico da angiotomografia em pacientes candidatos a transplante renal atraves da análise da região pélvica desses pacientes, a fim de verificar calcificações pré ateroscleróticas. 29 artigos foram inicialmente elegíveis neste estudo, resultando em 6 para análise após aplicação dos critérios de inclusão. Resultando em um total de 802 pacientes submetidos a CT (idade média 56 anos,63\% sexo masculino, cujas principais comorbidades eram diabetes mellitus, hipertensão arterial e tabagismo). Todos os estudos demonstraram alta acurácia no diagnóstico da calcificação por CT, nos quais a sensibilidade(S) e especificidade(E ) variaram $71 \%(S)$ e $73 \%(E)$ a $100 \%(S)$ e $92 \%(E)$. Em 4 estudos o transplante foi suspenso devido ao grau de calcificação (7,6\%), 2 artigos apresentaram relação de óbito com calcificação da região ilíaca e 5 artigos relataram perda do enxerto $(6,8 \%)$. Em todos os estudos primários a CT foi utilizada para planejamento cirúrgico com base no grau de calcificação $(25,2 \%$ de alterações no local de anastomose ou procedimentos vasculares).Os estudos divergiram quanto a relação da perda do enxerto e óbitos com a calcificação dos vasos ilíacos e a necessidade de suspensão do transplante com base no grau de calcificação. Os estudos que relataram relação direta com a calcificação tiveram, além dos resultados demonstrados, maior respaldo com as literaturas anteriores. Todos os artigos revelaram a importância da CT para identificar o melhor local para anastomose, desde o lado até o vaso a ser anastomosado, identificar a necessidade de intervenção pré cirúrgica como a endarterectomia, além de ser fator preditivo de complexidade cirúrgica.

Figura 1.Grafico comparativo dos desfechos pós transplante

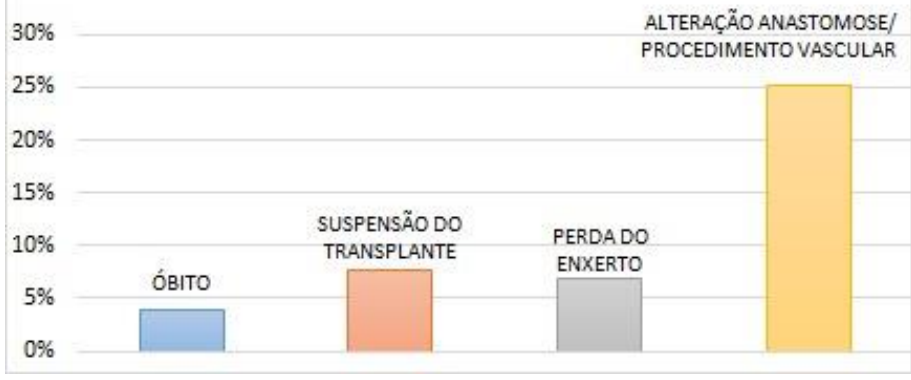

\section{Conclusões}

Concluímos que o uso da angiotomografia na avaliação de pacientes candidatos a terapia substitutiva renal apresentou grande valor na detecção de calcificação arterial em região pélvica, possibilitando identificar os melhores vasos para anastomose, permitindo indicações de procedimentos vasculares que evitariam a perda do enxerto, além da elevada acurácia em predizer complicações cirúrgicas.

1-Andres A, Revilla Y, Ramos A, et al. Helical computed tomography angiography is the most efficient test to assess vascular calcifications in the iliac arterial sector in renal transplant candidates. Transplant Proc 2003; 35:1682-1683

2- DAVIS, Bradley et al. Application of a Novel CT-Based Iliac Artery Calcification Scoring System for Predicting Renal Transplant Outcomes.American Journal of Roentgenology, [S. 1.], 2016. 